\title{
Case review of perinatal deaths at hospitals in Kigali, Rwanda: perinatal audit with application of a three-delays analysis
}

Aimable Musafili ${ }^{1,2^{*}}$, Lars-Åke Persson ${ }^{2}$, Cyprien Baribwira ${ }^{3}$, Jessica Påfs ${ }^{2}$, Patrick Adam Mulindwa ${ }^{4}$ and Birgitta Essén ${ }^{2}$

\begin{abstract}
Background: Perinatal audit and the three-delays model are increasingly being employed to analyse barriers to perinatal health, at both community and facility level. Using these approaches, our aim was to assess factors that could contribute to perinatal mortality and potentially avoidable deaths at Rwandan hospitals.

Methods: Perinatal audits were carried out at two main urban hospitals, one at district level and the other at tertiary level, in Kigali, Rwanda, from July 2012 to May 2013. Stillbirths and early neonatal deaths occurring after 22 completed weeks of gestation or more, or weighing at least $500 \mathrm{~g}$, were included in the study. Factors contributing to mortality and potentially avoidable deaths, considering the local resources and feasibility, were identified using a three-delays model.

Results: Out of 8424 births, there were 269 perinatal deaths (106 macerated stillbirths, 63 fresh stillbirths, 100 early neonatal deaths) corresponding to a stillbirth rate of 20/1000 births and a perinatal mortality rate of 32/1000 births. In total, 250 perinatal deaths were available for audit. Factors contributing to mortality were ascertained for $79 \%$ of deaths. Delay in care-seeking was identified in 39\% of deaths, delay in arriving at the health facility in 10\%, and provision of suboptimal care at the health facility in 37\%. Delay in seeking adequate care was commonly characterized by difficulties in recognising or reporting pregnancy-related danger signs. Lack of money was the major cause of delay in reaching a health facility. Delay in referrals, diagnosis and management of emergency obstetric cases were the most prominent contributors affecting the provision of appropriate and timely care by healthcare providers. Half of the perinatal deaths were judged to be potentially avoidable and $70 \%$ of these were fresh stillbirths and early neonatal deaths.

Conclusions: Factors contributing to delays underlying perinatal mortality were identified in more than threequarters of deaths. Half of the perinatal deaths were considered likely to be preventable and mainly related to modifiable maternal inadequate health-seeking behaviours and intrapartum suboptimal care. Strengthening the current roadmap strategy for accelerating the reduction of maternal and neonatal morbidity and mortality is needed for improved perinatal survival.
\end{abstract}

Keywords: Perinatal audit, Three-delays model, Urban hospitals, Rwanda

\footnotetext{
* Correspondence: aimable.musafili@kbh.uu.se

${ }^{1}$ Paediatric and Child Health Department, University of Rwanda, Kigali,

Rwanda

${ }^{2}$ Department of Women's and Children's Health, International Maternal and

Child Health (IMCH), Uppsala University, Akademiska Sjukhuset, Uppsala

SE-751 85, Sweden

Full list of author information is available at the end of the article
} 


\section{Background}

Around 4.8 million perinatal deaths occur each year in the world, and, of these, 98\% take place in low- and middle-income countries $[1,2]$. Perinatal deaths consist of stillbirths or fetal deaths from 22 weeks of gestation and early neonatal deaths or deaths within the first week after birth [3]. Perinatal mortality is regarded as a key indicator, reflecting the quality of healthcare provided to women during pregnancy and childbirth as well as to neonates in the first week of life [4].

Perinatal audit and feedback is a clinical approach widely utilized to improve quality of care and to reduce perinatal mortality [5]. The audit process provides an opportunity to learn from critical events in the management of care of pregnant women and neonates. Healthcare workers are prompted to change their routines once they are given feedback about the inadequate practices that lead to these events [6]. Perinatal audit also explores factors occurring at home or in the community that were likely to contribute to the outcome. A metaanalysis of studies on perinatal audit conducted in lowand middle-income countries suggested that up to $30 \%$ of perinatal deaths could be averted after the implementation of a perinatal audit [7].

The three-delays model is considered to be a suitable framework for identifying and assessing the barriers faced by pregnant women before they access appropriate care. This approach was initially conceived to explore factors leading to maternal deaths. Three categories of factors are identified: delay in making decisions in seeking care (phase-one delay), delay in reaching the health facility (phase-two delay), and delay in receiving appropriate care at the health facility (phase-three delay) [8]. This model has also been applied to understand factors related to perinatal and neonatal mortality $[9,10]$.

In 2012, the rate of perinatal mortality at public health institutions in Rwanda was 29/1000 births [11]. According to the Demographic and Health Survey conducted in 2015, almost all women (99\%) attended antenatal care. Among them, 44\% attended four times or more. The proportion of women giving birth at health facilities was 91\% [12].

In 2009 , the maternal mortality audit process was implemented and routinely carried out at hospital level throughout Rwanda [13]. Two years later, the neonatal mortality audit process was also integrated into hospital practices [14]. However, a perinatal audit is not yet routinely performed. There is a need to also focus on factors related to fresh stillbirths as most of these are similar to the factors contributing to early neonatal deaths in this context [15]. Further, obstetric complications leading to maternal deaths are also common causes of perinatal deaths [16]. Thus, performing a perinatal audit may help to link knowledge between both the maternal and neonatal mortality audits.
The aim of this study was to carefully monitor births and outcomes (macerated or fresh stillbirths, live births, early neonatal deaths) at two Kigali hospitals, to identify factors causally related to perinatal deaths and to relate these to the three-delays factors experienced by delivering women; (1) mother's inadequate healthcareseeking behaviour, (2) difficulties in reaching a health facility, or (3) receipt of suboptimal medical and nursing care at the health facility, and to assess whether these deaths were potentially preventable.

\section{Methods \\ Study design}

The study adopted a perinatal audit design [4]. The audit was conducted prospectively and was related to the cases of perinatal deaths that had occurred among babies born in two urban hospitals.

\section{Study setting}

This study was part of a programme that was aimed at analysing the causes and determinants of perinatal mortality at a district hospital $(\mathrm{DH})$ and a tertiary referral hospital (TRH) from 18 July 2012 to 8 May 2013. These two hospitals, located in Kigali, Rwanda, assisted in almost 7900 and 2200 deliveries in 2012, respectively. The $\mathrm{DH}$ conducts the most deliveries in the country, whereas the TRH serves as the largest referral and University teaching hospital.

During the study period, the midwifery, nursing and medical teams working in the maternity and neonatal units included 79 midwives and nurses, 14 general practitioners, 3 obstetricians, and 2 paediatricians in the $\mathrm{DH}$, whereas there were 78 midwives and nurses, 4 obstetricians, 2 paediatricians, 5 residents in paediatrics and 15 in obstetrics and gynaecology in the TRH. Most patients received in the two hospitals lived in the capital, which had approximately 1200000 inhabitants in 2012. Other patients came from the surrounding areas of the city or were referred from other urban or rural health facilities.

Patients attending both hospitals were mainly covered by the community health insurance system, as shown in our previous study [17]. Patients usually followed referral steps starting from public health centres or posts, then to district hospitals, and finally, to tertiary level hospitals. Those who bypassed these referral pathways were not covered by the community health insurance system, except in emergencies. Other existing health insurance systems cover civil servants, the military, and people working in the private sector. These insurance systems frequently covered patients who attended the TRH. Patients who had no insurance cover needed to pay all hospital charges themselves. 


\section{Study population}

Stillbirths and early neonatal deaths, which together constitute perinatal deaths, among births at the two hospitals were consecutively included in the study. A stillbirth was defined as a fetal death after at least 22 completed weeks of gestation or weighing $500 \mathrm{~g}$ or more at birth. An early neonatal death was defined as a live birth dying within the first seven days of life, the early neonatal period, and born after at least 22 completed weeks of gestation or weighing $500 \mathrm{~g}$ or more at birth [18]. Deaths that occurred among babies born outside the study sites were excluded from the study.

\section{Data collection}

In each hospital, one nurse, one midwife, and one doctor were recruited and trained over two days on the study's aim and data collection procedures, and how to conduct interviews with bereaved mothers and healthcare providers involved in the study using a questionnaire containing both closed and open-ended questions.

The first part of the questionnaire included information on the socioeconomic characteristics of the mothers and their households, such as household assets, maternal education and place of residence, and health insurance coverage, as well as the demographic characteristics of the mothers and their babies, such as parity, maternal and gestational age, birth weight, and sex. The second part included data on routine antenatal screening and care, pregnancy complications and their management, characteristics of labour and delivery, condition of the baby at birth (alive or stillbirth) and afterwards, Apgar score, management of the newborn's illness that led to a fatal outcome, and time and causes of death.

A fetus that died during labour or delivery was defined as a fresh stillbirth. These stillbirths were also labelled intrapartum-related stillbirths as most of them were due to intrapartum-related insults or injuries [19]. When a death occurred before the onset of labour or delivery with signs of a degenerative process, the fetus was classified as a macerated stillbirth [18]. The time of fetal death in relation to admission was also recorded.

The third part of the questionnaire comprised information obtained from interviews with mothers and healthcare providers. Mothers were encouraged to describe the chain of events leading up to the death, including both clinical and social elements, such as reasons for seeking care at a health facility, the onset and development of symptoms, actions taken, barriers encountered when seeking care, and the clinical attention received at the health facility. Important dates and time-related events were recorded. When mothers were not available, information was sought from their partners or closest relatives. Healthcare providers also provided additional narratives about the circumstances that led to the death.

Data collected through interviews were systematically cross-checked against the information extracted from clinical records. When discrepancies were noted, interviews were repeated or other information sources were re-checked. Random repeat interviews were regularly performed to reinforce the quality of the data.

Narratives summarizing the information collated from the different sources were prepared for each case of death by the main investigator. The actual data collection was preceded by one month of piloting of the study procedures. The main investigator rigorously supervised the fieldwork, assisted by one doctor from each hospital.

\section{Audit meetings}

Members of the audit committee were recruited from the hospitals and included two obstetricians, two paediatricians, and the main investigator, who is also a paediatrician. The main investigator introduced the study objectives and procedures at the first meeting. He also presented all cases of perinatal deaths that were discussed in each session, which was scheduled according to the availability of the members.

These presentations were based on the individual narratives and were followed by open discussions to identify the causes of the deaths, the underlying factors, and any potentially avoidable deaths. A single cause of death was assigned to each case. The avoidable factors were classified based on the three-delays model [8]. In some instances, several delays were identified for an individual case. The discussions were aimed at evaluating whether the delay had possibly or likely contributed to the fatal outcome. This process was consistent with previous evaluation studies completed elsewhere [4].

The quality of care provided to mothers and neonates was judged against evidence-based practices, as expressed in local guidelines and protocols or in the scientific literature [20]. The adequacy of care was also related to feasibility and the availability of resources and opportunities, such as any investigations in making a diagnosis, and the availability of drugs, consumables, equipment, and infrastructure.

A death was considered avoidable if improved management using available resources and opportunities would have altered the outcome. This approach has been applied in other reports on perinatal audit in low- and middle-income countries [21, 22]. In addition, the timing of death in relation to the mother's admission to the hospital provided important orientation regarding the prevention of death. Audible fetal heartbeat on admission increased the likelihood that death would have been avoided.

The auditors discussed each case to reach a consensus. In case of disagreement, all sources of information were 
rechecked and conclusions were made during the subsequent meeting. The senior obstetrician had the final word in matters of pregnancy, labour, and deliveryrelated events, while the paediatrician made the final decision regarding neonatal care and mortality [23].

\section{Data analysis}

The socio-demographic and clinical characteristics of the mothers and their babies included in the study were summarised in descriptive tables. Based on the individual narrative stories for each case of death, the auditors assessed the contributing factors that could be attributed to the three delays as well as to potentially avoidable deaths. Factors contributing to the same phase of delay were grouped together and listed in descriptive tables. Each phase of delay was illustrated by a case study that described barriers to access adequate maternal and neonatal care. Potentially avoidable deaths and their frequencies were described. IBM SPSS Statistics version 20 (IBM Corporation, Armonk, NY) was used for descriptive statistics.

The Rwanda National Ethics Committee granted ethical approval for this study (ethics approval no. 086 RNEC/2012, Kigali, Rwanda).

\section{Results}

\section{Characteristics of participants}

Out of 8424 births, there were 269 perinatal deaths (106 macerated stillbirths, 63 fresh stillbirths, 100 early neonatal deaths) corresponding to a stillbirth rate of 20/ 1000 births and a perinatal mortality rate of 32/1000 births (Fig. 1). Singleton and twin births constituted 94 and $6 \%$ of the cases, respectively. Nineteen cases of deaths were not reviewed due to lack of information as the mothers had been discharged $(n=14)$ or died $(n=2)$ before interviews or refused to participate in the study $(n=2)$. One neonate died shortly after referral to another hospital and was also excluded from the study.

On admission, fetal heartbeats were inaudible for all macerated stillbirths and audible in $40 \%$ of the fresh stillbirths.

Table 1 shows the characteristics of mothers, pregnancies, deliveries, and children among the cases of perinatal death that occurred during the study period. Most babies were born to women living in urban areas and having a primary educational level. Most babies were born to women living with partners. The median maternal age was 28 years (range 16-47). Most women were multipara (parity $1-4$ ). Most babies were born to women who had attended at least one antenatal care visit and one-fifth were born to mothers who had attended the recommended four antenatal care visits. Vaginal delivery was the most frequent mode of delivery. The highest proportions of deaths (53\%) consisted of preterm births.

Maternal morbidity during pregnancy and labour is summarized in Table 2. Most babies, that is to say, 156/ 250 (62\%), were born to mothers who had no complications during pregnancy. The most common medical problems identified in the remaining mothers were hypertensive disorders (preeclampsia, eclampsia, and pre-existing hypertension) and HIV/AIDS.

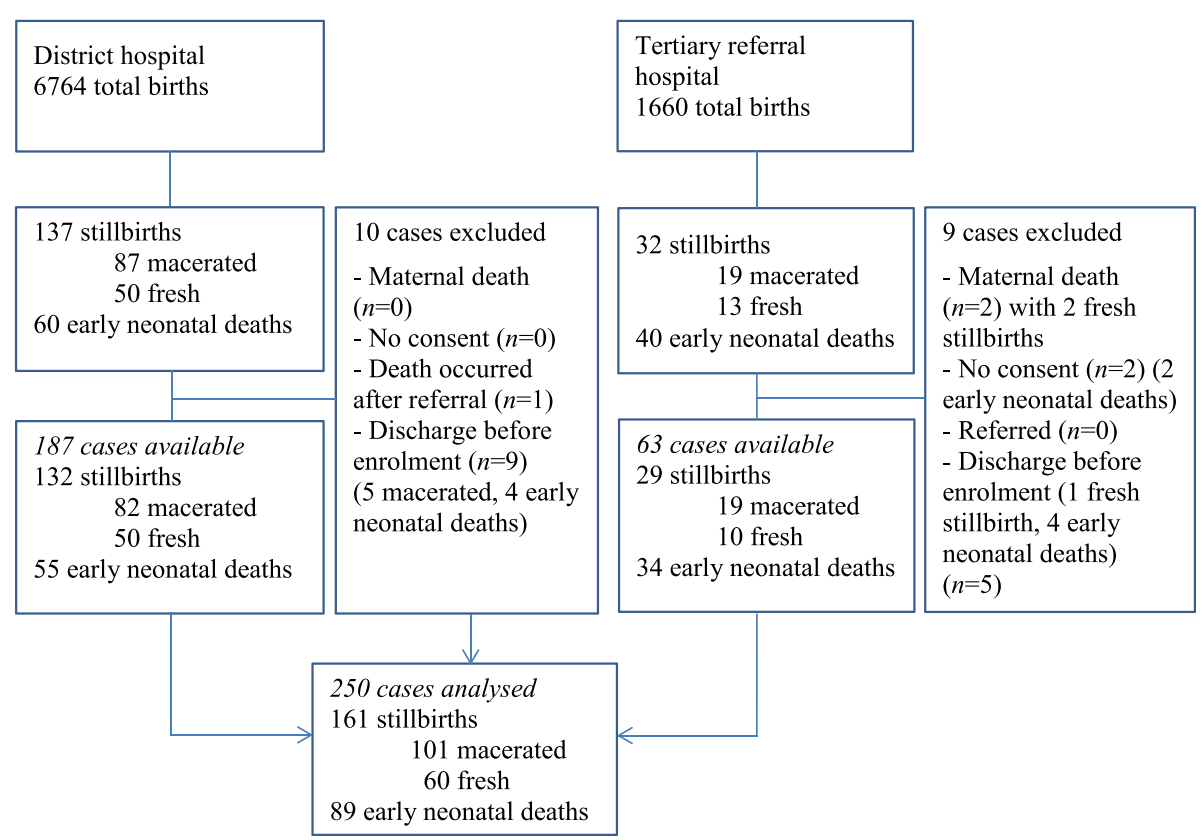

Fig. 1 Flowchart of study population selection 
Table 1 Maternal, pregnancy, and childbirth characteristics for the cases of perinatal deaths at two hospitals in Kigali, Rwanda July 2012-May 2013

\begin{tabular}{|c|c|c|}
\hline \multirow[t]{2}{*}{ Characteristics } & \multicolumn{2}{|c|}{ Perinatal deaths } \\
\hline & $n$ & $\%$ \\
\hline \multicolumn{3}{|l|}{ Residence } \\
\hline Urban & 225 & 90 \\
\hline Rural & 25 & 10 \\
\hline \multicolumn{3}{|l|}{ Maternal education } \\
\hline Secondary or higher & 91 & 36 \\
\hline Primary & 142 & 57 \\
\hline No formal education & 17 & 7 \\
\hline \multicolumn{3}{|l|}{ Marital status } \\
\hline Living with partner & 206 & 82 \\
\hline Living without partner & 44 & 18 \\
\hline \multicolumn{3}{|l|}{ Maternal age at childbirth (years) } \\
\hline$<20$ & 14 & 6 \\
\hline $20-34$ & 182 & 73 \\
\hline$>34$ & 54 & 21 \\
\hline \multicolumn{3}{|l|}{ Parity } \\
\hline 0 & 106 & 42 \\
\hline $1-4$ & 125 & 50 \\
\hline$>4$ & 19 & 8 \\
\hline \multicolumn{3}{|l|}{ Gestational age (weeks) } \\
\hline $22-27$ & 40 & 16 \\
\hline $28-33$ & 63 & 25 \\
\hline $34-36$ & 31 & 12 \\
\hline$>36$ & 116 & 47 \\
\hline \multicolumn{3}{|l|}{ Antenatal visits } \\
\hline 0 & 15 & 6 \\
\hline $1-3$ & 188 & 75 \\
\hline$>3$ & 47 & 19 \\
\hline \multicolumn{3}{|l|}{ Mode of delivery } \\
\hline Spontaneous vaginal delivery & 170 & 68 \\
\hline Caesarean section & 76 & 30 \\
\hline Vacuum-assisted delivery & 4 & 2 \\
\hline
\end{tabular}

The causes of perinatal mortality were categorized according to the type of death and phase of delay identified during the audit meetings (Table 3). For most cases of macerated as well as fresh stillbirths the cause of death was unknown. Among the suspected causes, asphyxia due to maternal hypertensive disorders (preeclampsia, eclampsia, and pre-existing hypertension) was the most common, followed by malformations for macerated stillbirths and umbilical cord prolapse or looping around the neck for fresh stillbirths. The major causes of early neonatal deaths were intrapartum-related neonatal
Table 2 Maternal morbidity during pregnancy and labour among the cases of perinatal death at two hospitals in Kigali, Rwanda July 2012- May 2013

\begin{tabular}{|c|c|c|}
\hline \multirow{2}{*}{$\begin{array}{l}\text { Maternal diseases and } \\
\text { complications }\end{array}$} & \multicolumn{2}{|c|}{ Perinatal deaths } \\
\hline & $n$ & $\%$ \\
\hline Hypertensive disorders $^{a}$ & 40 & 16 \\
\hline HIV/AIDS & 30 & 12 \\
\hline Bleeding $^{b}$ & 18 & 7 \\
\hline Chorioamniotitis/sepsis & 7 & 3 \\
\hline Diabetes mellitus ${ }^{c}$ & 4 & 2 \\
\hline Uterine rupture & 4 & 2 \\
\hline Malaria & 2 & 1 \\
\hline Severe anaemia & 1 & 0 \\
\hline Others $^{d}$ & 3 & 1 \\
\hline All & 94 & 38 \\
\hline None & 156 & 62 \\
\hline Total & 250 & 100 \\
\hline \multicolumn{3}{|c|}{$\begin{array}{l}\text { Note that the total of individual diseases/complications }(n=109) \text { was higher } \\
\text { than that of deaths }(n=94) \text { because women could have } \\
\text { several diseases/complications } \\
\text { a Hypertensive disorders: preeclampsia }(n=32) \text {, pre-existing hypertension ( } n= \\
\text { 5) eclampsia }(n=3) \\
\text { b Bleeding: placenta abruption }(n=13) \text {, placenta praevia }(n=4) \\
\text { c Diabetes mellitus: gestational }(n=3) \text { or pre-existing }(n=1) \\
\text { d Others: Asthma }(n=2) \text {, cardiac disease }(n=1)\end{array}$} \\
\hline
\end{tabular}

causes, followed by prematurity or low birth weight and neonatal infections.

Of the women whose pregnancies ended in macerated stillbirths, most had experienced a phase-one delay, whereas those who had fresh stillbirths or early neonatal deaths more frequently had a phase-three delay. A phase-two delay was less common in all groups of perinatal deaths.

\section{Factors contributing to delays}

Potentially avoidable factors related to the three delays were identified in 79\% (197/250) of perinatal deaths. Several factors could contribute to one case of death. Thus, the total number of factors contributing to mortality was higher than that of deaths (Table 4).

\section{Delay in seeking appropriate care: Phase-one delay}

As shown in Table 4, phase-one delay (delay in decisionmaking to seek appropriate care) was more frequent than other phases of delay and was identified in $39 \%$ $(98 / 250)$ of deaths. The most prominent reason for this delay was failure to recognize or report danger signs that occurred during pregnancy or labour. This delay was identified in 81 cases. The most frequently reported danger sign consisted of poor or absent fetal movements and signs of preterm labour such as preterm contractions or preterm rupture of membranes. 
Table 3 Causes of perinatal mortality according to the type of perinatal death and phase of delay identified during audit meetings at two hospitals in Kigali, Rwanda July 2012- May 2013

\begin{tabular}{|c|c|c|c|c|c|}
\hline \multirow[t]{2}{*}{ Causes of deaths } & \multicolumn{2}{|c|}{ Perinatal deaths } & \multirow{2}{*}{$\begin{array}{l}\text { Cases with } \\
\text { phase-one delay }\end{array}$} & \multirow{2}{*}{$\begin{array}{l}\text { Cases with } \\
\text { phase-two delay }\end{array}$} & \multirow{2}{*}{$\begin{array}{l}\text { Cases with } \\
\text { phase-three delay }\end{array}$} \\
\hline & $n$ & $\%$ & & & \\
\hline Total & 250 & 100 & 98 & 24 & 93 \\
\hline \multicolumn{6}{|l|}{ Causes of macerated stillbirths } \\
\hline Hypertensive disorders $^{d}$ & 11 & 11 & 7 & 3 & 8 \\
\hline Malformations & 6 & 6 & 0 & 0 & 0 \\
\hline $\begin{array}{l}\text { Preterm pre-labour rupture } \\
\text { of membranes }\end{array}$ & 5 & 5 & 3 & 1 & 2 \\
\hline Placenta abruption & 2 & 2 & 0 & 0 & 1 \\
\hline External trauma & 1 & 1 & 1 & 0 & 0 \\
\hline Unknown & 76 & 75 & 62 & 3 & 7 \\
\hline Total & 101 & 100 & 73 & 7 & 18 \\
\hline \multicolumn{6}{|l|}{ Causes of fresh stillbirths } \\
\hline Hypertensive disorders ${ }^{d}$ & 11 & 18 & 5 & 2 & 9 \\
\hline $\begin{array}{l}\text { Umbilical cord prolapse/loop } \\
\text { around neck }\end{array}$ & 10 & 17 & 3 & 1 & 4 \\
\hline Placenta abruption & 7 & 12 & 1 & 1 & 3 \\
\hline Prolonged labour/obstructed labour & 6 & 10 & 0 & 0 & 2 \\
\hline Uterine rupture & 4 & 7 & 0 & 1 & 3 \\
\hline Malformations & 3 & 5 & 0 & 0 & 0 \\
\hline $\begin{array}{l}\text { Preterm pre-labour rupture } \\
\text { of membranes }\end{array}$ & 3 & 5 & 1 & 2 & 2 \\
\hline Unknown & 16 & 26 & 3 & 1 & 10 \\
\hline Total & 60 & 100 & 13 & 8 & 33 \\
\hline \multicolumn{6}{|l|}{ Causes of early neonatal deaths } \\
\hline Intrapartum-related neonatal deaths & 34 & 38 & 4 & 2 & 26 \\
\hline Prematurity/Low birth weight & 30 & 34 & 5 & 5 & 12 \\
\hline Infections & 11 & 12 & 3 & 2 & 2 \\
\hline Malformations & 8 & 9 & 0 & 0 & 0 \\
\hline Neonatal jaundice & 1 & 1 & 0 & 0 & 1 \\
\hline Unknown & 5 & 6 & 0 & 0 & 1 \\
\hline Total & 89 & 100 & 12 & 9 & 42 \\
\hline
\end{tabular}

Note that each perinatal death could have had more than one type of delay

a Phase-one delay: delay in decision-making and seeking adequate care

b Phase-two delay: delay in reaching health facility

c Phase-three delay: delay in receiving appropriate care at health facility

${ }^{d}$ Hypertensive disorders: preeclampsia, eclampsia, pre-existing hypertension

Some women were not aware of the severity of the danger signs and preferred to stay at home waiting for spontaneous resolution of these problems. Others had asked for advice from a partner, relative, neighbour or friend or had sought help from private pharmacies or traditional healers. The decision to seek care at health facilities had often been taken late, when danger signs had worsened or failed to subside.

Five women, who sought informal care providers, received traditional medicines because the main cause of danger signs was attributed to ifumbi. This was vaguely described as a clinical condition with abnormal symptoms varying from simple discomfort to major complications. The users of traditional medicines believed that ifumbi could be effectively cured by traditional medicine.

Four women had also used traditional medicines during labour, both before and after admission. The women considered these as effective stimuli of uterine contractions. Some also believed in the potential protection of these medicines against any witchcraft that could negatively influence maternal and fetal outcomes. Case study 
Table 4 Delays experienced by pregnant women and/or their babies in accessing appropriate care at health facility, as reported by the panellists during perinatal audit at two hospitals in Kigali, Rwanda July 2012-May 2013

\begin{tabular}{|c|c|}
\hline Delays & Cases with delays \\
\hline Phase-one: care-seeking & 98 \\
\hline Lack of recognition of danger signs & 81 \\
\hline Poor uptake of or compliance to formal care & 25 \\
\hline Phase-two: reaching health facility & 24 \\
\hline Lack of money & 15 \\
\hline Long distance & 6 \\
\hline Lack of health insurance & 3 \\
\hline Social or family reasons & 3 \\
\hline Phase-three: quality of care at health facility & 93 \\
\hline \multicolumn{2}{|l|}{ Before admission } \\
\hline No or late diagnosis & 9 \\
\hline \multicolumn{2}{|l|}{ Inadequate management } \\
\hline Delay in the referral ${ }^{a}$ & 13 \\
\hline Inadequate care or monitoring & 12 \\
\hline \multicolumn{2}{|l|}{ After admission } \\
\hline Late diagnosis & 3 \\
\hline \multicolumn{2}{|l|}{ Inadequate management } \\
\hline Inadequate monitoring or late intervention & 41 \\
\hline Non-adherence to guidelines or best practices & 5 \\
\hline \multicolumn{2}{|l|}{ After delivery } \\
\hline \multicolumn{2}{|l|}{ Inadequate management } \\
\hline Insufficient care & 11 \\
\hline Inadequate monitoring & 3 \\
\hline Total & 197 \\
\hline
\end{tabular}

a The delays in referrals were observed when women were referred either from a health centre or private clinic to the district hospital or from a private clinic or the district hospital to the tertiary referral hospital. Note that there were more factors contributing to mortality than cases of deaths because several factors categorized as the same or different phases of delay could be identified in one case of death

A (Additional file 1) illustrates a situation where the lack of knowledge on the severity of danger signs, combined with the utilization of traditional medicines, contributed to a phase-one delay.

Poor uptake of care by registered healthcare providers was noted in 16 cases. In two cases, the pregnancies were reportedly unintended and no antenatal care was sought. In two other cases, women were single mothers and did not have time to seek antenatal care due to a heavy workload to earn their living. Six women, including two with poor obstetrical histories (previous perinatal death), did not attend follow-up visits during pregnancy. Three of them explained that they could not return because they had not been clearly briefed by the healthcare provider about the need to attend. Others failed to give clear reasons for their non-attendance at follow-up visits. Five women initially wanted to deliver at home and came late to the health facility, when complications had already occurred. One woman refused the induction of labour suggested by her doctor despite having preterm rupture of the membranes and oligohydramnios.

\section{Delay in reaching a health facility: Phase-two delay}

The factors related to phase-two delay - when a woman decided to seek care but was delayed in reaching a health facility due to various reasons, such as lack of transport or limited revenue - were found in 10\% (24/ 250) of cases (Table 4). Women reported lack of money as a key problem that delayed their timely care-seeking. They mainly expressed difficulties in mobilising money to cover the cost of transport or other expenses not covered by health insurance during their hospital stay. An example of delay that was ascribed to the lack of money is provided in case study B (Additional file 2).

Long travel distance (with a mean duration of $90 \mathrm{~min}$ ) from home to the health facility or between health institutions was also reported in six cases of perinatal death. For four women, the long distance was compounded by a long walk or their having to travel by bike or motorbike on roads with poor conditions. For three women, the process of renewing or purchasing health insurance was undertaken late, which resulted in a delay to reach the health facility. Delayed access to care also occurred for two women because of illness in the family. One woman did not want to seek care during the night because it would not be convenient for her.

\section{Delay in receiving appropriate care at a health facility: Phase-three delay}

Various circumstances that led to the phase-three delays in relation to suboptimal care received once at a health facility - were found in 37\% (93/250) of deaths, as shown in Table 4.

Before admission, most cases of death were related to delays in women's referrals from health centres or private clinics to district hospitals or from the district to the tertiary referral hospital. Such delays were identified in 12 cases, where death was subsequent to intrapartumrelated hypoxia, preterm rupture of membranes, or preterm labour. One of these deaths was related to uterine rupture and occurred at a health centre after prolonged labour. In another case, a woman who had signs of preterm labour reportedly had a long waiting time $(4 \mathrm{~h})$ at a health centre before being examined and referred to the district hospital.

Late diagnosis and failure to diagnose or perform proper management were other common inadequacies in the practices of healthcare providers. In five cases of poor obstetric history (previous perinatal death), these 
women did not receive any special attention during pregnancy. In one case, gestational diabetes was diagnosed late in the pregnancy by providers at a district hospital in a woman who had initially been attending antenatal care clinics at a health centre. In another case, a woman referred from a health centre for poor progress of labour also had an umbilical cord prolapse, which was detected upon arrival at the hospital.

Similar delays in diagnosis or management had occurred for some women presenting with hypertension or preeclampsia. Another woman who also had gestational diabetes had received insufficient treatment at a private clinic. Substandard provision of care was observed in another woman at 37 weeks of gestation who was treated by a nurse at a health centre with an anti-inflammatory drug for one week due to multiple joint pain. Another case was related to umbilical cord prolapse that occurred at a health centre after an amniotomy was performed.

After admission, insufficient labour and fetal monitoring, and delay in taking appropriate action during the management of pregnancy or labour complications were the most frequent factors related to the phase-three delay. These inadequacies were found in 40 cases where the deaths were linked to various conditions, mainly prolonged or obstructed labour, preterm labour, maternal hypertension, preeclampsia and placenta abruption. These conditions also included two cases of uterine rupture that occurred in hospital. There were difficulties in handling one case of breech presentation and another case of vacuum-assisted delivery. In both cases, the doctor was called to assist after unsuccessful efforts by a midwife or nurse to perform the delivery. A caesarean section was delayed for $30 \mathrm{~min}$ because two other caesarean sections were ongoing and no other operating theatre was available in the hospital.

Non-adherence to the national guidelines was another barrier that delayed access to appropriate care in hospital after admission. An accidental double-dose of intravaginal misoprostol $(100 \mathrm{mcg})$ was repeatedly administered to two women for labour induction. This could have led to the hyper-stimulation of uterus and fetal heart, causing or aggravating fetal hypoxia, which was the cause attributed to the death.

An attempt at vaginal delivery of a twin pregnancy was made in another woman, where ultimately a caesarean section was urgently performed due to the failure of delivery by vaginal route, which subsequently caused fetal asphyxia. An early caesarean section would have prevented this asphyxia, because the first twin had presented in a breech position. The national guidelines suggest a caesarean where the first twin has not presented in a cephalic position.

Late diagnosis of some pregnancy-related complications was another contributing factor to the phase-three delay, which was identified in two cases of placenta abruption and one case of preeclampsia. In these cases, the delay in making the diagnoses also affected the initiation of adequate treatment, as depicted in case study C (Additional file 3).

After delivery, insufficient newborn resuscitation and management of neonates with severe intrapartumrelated hypoxia constituted major causes of suboptimal care. In some instances, resuscitation was not promptly initiated within the first minute after birth.

Some equipment, including suction tubes and facemasks for ventilation, were used inappropriately or were not in good condition when resuscitation began. In two cases, ventilation with bag and mask was applied in two neonates before proper skin drying and stimulation. Upon arrival to the neonatal intensive care unit, they were still wrapped in the wet cloths used when drying the skin at birth. In one of these cases, the endotracheal intubation was delayed due to the limited skills of the healthcare provider who was performing the advanced resuscitation.

The management of some cases of intrapartum-related hypoxia was temporarily hampered by a lack of secondline treatment such as phenytoin. Some of these cases also required the use of a ventilator machine, equipment that was not available in one of the two study hospitals. Two cases of death occurred while the transfer of the infant to a higher level of care was being discussed.

Another important suboptimal care factor consisted of the irregular monitoring of vital signs. This was observed in three preterm babies who developed hypothermia and bradycardia. In the three remaining cases, suboptimal care factors were related to inadequate management of some neonatal emergency conditions, including jaundice, feeding of a sick preterm baby, and delay in blood transfusion. The jaundice had eventually evolved into kernicterus. The inappropriate enteral feeding of one preterm baby presenting with food intolerance and frequent vomiting led to pulmonary aspiration.

\section{Avoidable deaths}

Of 250 perinatal deaths reviewed, $51 \%$ were considered likely avoidable and $70 \%$ of them were fresh stillbirths and early neonatal deaths. When considering fresh stillbirths and early neonatal deaths alone, $60 \%$ of them were potentially preventable.

\section{Discussion}

\section{Main findings}

This perinatal audit revealed that a number of factors contributed to delays, which, in total, were identified in more than three-quarters of perinatal deaths. The most frequent delays were related to inadequate maternal care-seeking during pregnancy and childbirth (phaseone delay), followed by suboptimal care factors that 
occurred at health facilities (phase-three delay). Barriers encountered after deciding to go to a health facility (phase-two delay) were less common. Delays in seeking formal care were mainly due to the lack of recognition of danger signs. Delays in referrals, poor labour and fetal monitoring, and delayed action during the management of obstetric emergencies were the most predominant factors assessed at health facilities. Lack of money was the most common reason for not reaching the health facility in time. Approximately half of the perinatal deaths were judged to be potentially avoidable and most of these were fresh stillbirths and early neonatal deaths.

\section{Strengths and limitations}

This perinatal audit study was the first of this kind carried out in Rwanda and included two main urban hospitals, which together manage a high number of complicated pregnancies. The risk of recall bias by mothers or healthcare providers was judged to be low, given that they were interviewed shortly after delivery or neonatal death, and before discharge from hospital.

The mothers included in this study predominantly lived in urban settings, mainly in the capital. Thus, the results may not be generalizable to the country as a whole due to varying social and health services conditions.

Making a distinction between miscarriage and stillbirths could be difficult, especially when the last menstrual period was unknown. We tried to minimize this bias by applying the standardized definition of $500 \mathrm{~g}$ as the lower limit of birth weight for stillbirths. Ultrasound findings were also used for dating pregnancies. Most women with suspected intrauterine fetal deaths underwent this examination to confirm death and to assess whether there was any visible malformation, and, in addition, gestational age and estimated weight were obtained at this time.

Another potential bias was the misclassification of fresh stillbirths as early neonatal deaths. However, this bias was minimal because each birth was attended by a skilled healthcare provider, who also assessed the signs of life after delivery [24].

No autopsy was performed to further investigate the cause of death. This implies that the number of deaths attributed to congenital malformations may in fact be higher. The classification of lethal congenital malformations were made by clinical examination, and all $(n=17)$ were considered non-avoidable [25].

Our investigation was, in principle, limited to data that were available at the two study hospitals. We were not able to access detailed information from other healthcare providers or facilities that had been involved in the management of the pregnant and birthing mothers. This is a limitation in that we were not able to obtain a full description of the quality of care provided to the women before they were admitted to the study hospitals.

\section{Comparison with other findings}

The particularity of this study was the application of a perinatal audit with a three-delays model to explore factors underlying mortality in all perinatal deaths, regardless of age or weight at birth. Recently, a study carried out in one regional hospital in Tanzania employed the same approach but the study population was restricted to perinatal deaths weighing $2000 \mathrm{~g}$ or more [9]. Many other investigators in low-, middle- and high-income countries have limited the audit either to suboptimal care factors occurring at a health facility $[26,27]$ or to specific groups of perinatal deaths based on gestational age or birth weight [23, 28-30].

The factors related to the three phases of delay in accessing care were identified in $79 \%$ of all deaths in this study. This proportion is similar to that $(80 \%)$ found by authors in Tanzania during a perinatal audit performed at a tertiary referral hospital [29]. These figures are higher than those reported from studies in 10 European countries, the Netherlands, and Uganda, where the factors contributing to mortality were assessed to be found in 46,32 and $20 \%$ of deaths, respectively [4, 27, 30]. However, the latter three studies had applied different methodologies and definitions as compared to our study. The two studies conducted in the Netherlands and Uganda were also restricted to suboptimal care factors occurring at health institutions. In addition, the quality of care in each country or setting and the expert panel involved in the perinatal audit may also explain the different proportions of factors contributing to perinatal mortality. Evidence has shown that the levels of agreement on suboptimal care factors may vary between auditors $[29,31]$.

Mothers' delay in decision-making to seek appropriate care was assessed as the most common contributing factors to perinatal mortality, followed by suboptimal care at hospital. However, the frequency of these delays was not consistent across the three groups of perinatal deaths (Table 3). Phase-one delay (care-seeking) was the most frequent in the group of macerated stillbirths, whereas phase-three delay (substandard care practices) was predominant among the groups of fresh stillbirths and early neonatal deaths.

An important reason to delay care-seeking was a lack of recognition of pregnancy or labour danger signs as well as social conditions, such as living without partner, unintended pregnancy, and misunderstandings between healthcare seeker and provider. These social stigmarelated factors affecting the timing of presentation to skilled care before or during labour were also found in 
earlier studies carried out in low- and middle-income countries [22, 32, 33].

Barriers could be worsened by cultural beliefs, which might prompt women to seek care from traditional healers and misuse traditional medicines, as seen in our study. Studies conducted in Nigeria on stillbirth and in Uganda on uterine rupture showed that the intake of traditional medicines reportedly had a detrimental effect on maternal and fetal health $[34,35]$.

Our research indicated that phase-two delay (reaching health facility) was less common than other delays. The plausible explanation for this result was that most women included in the study (90\%) lived in urban settings, where transport and health facilities were readily available. However, some issues, mainly lack of money and, less common, a long distance from home to health facility or between health facilities, lack of insurance and other social constraints, were linked to the phase-two delay. We have previously shown that lack of health insurance was associated with increased risk of perinatal deaths [17].

Studies in Uganda on neonatal mortality and in India on stillbirths and neonatal deaths showed that barriers to access the health facility were less common. These barriers included lack of transport, financial-related issues, lack of autonomy in decision-making in the absence of a partner, and a lack of guardians for children when mothers were not at home $[10,33]$. In many other low- and middleincome countries, long distances, poor roads, lack of transport, lack of financial means, particularly in poor households, and lack of insurance have also been listed as key barriers to reach health facilities [36-39].

A recent systematic review on avoidable factors contributing to maternal and perinatal mortality in lowincome settings showed that substandard practices by healthcare providers were the first contributing factors to death, followed by patient-related delays [22]. These findings are consistent with those reported in many other studies on perinatal audit [23, 29, 31]. A number of studies in these countries $[9,23,40]$ have further highlighted the pivotal contribution of substandard care to intrapartum-related stillbirths and early neonatal deaths, which also was the case in our study.

Delay in referral from lower to higher levels of care [33], inadequate antenatal care, delay of diagnosis, poor management of high-risk pregnancy $[7,27,34,41]$, inadequate labour and fetal monitoring $[9,23,29,32]$, and insufficient newborn resuscitation and management of preterm births $[15,30]$ have frequently been reported as major contributors to suboptimal care by healthcare providers. These factors were also similar to those identified in our study.

The audit committee in this study concluded that almost half of the perinatal deaths reviewed could have been prevented. Several studies on perinatal audit conducted around the world have shown that the proportions of potentially avoidable deaths could vary between 14 and $75 \%[28,29,42,43]$. The differences in preventable deaths observed across these studies could be attributed to the levels of mortality or quality of care in each setting as well as the definitions and methods applied.

This study has shown that $60 \%$ of fresh stillbirths and early neonatal deaths could have been averted. This proportion falls in between those reported by internal (58\%) and external (83\%) auditors in a similar study conducted in Sudan [31]. In another audit conducted in Tanzania, the proportion of fresh stillbirths and early neonatal deaths considered avoidable was estimated at $64 \%$ by internal auditors versus $79 \%$ by external auditors [29]. However, in the latter study, the audit was performed on deaths weighing at least $1500 \mathrm{~g}$.

\section{Policy implications}

The proportion of potentially avoidable deaths was relatively high, particularly among fresh stillbirths and early neonatal deaths, suggesting that there is considerable room for improved care and perinatal survival. However, in order to better assess the potential for improvement regarding the quality of care and perinatal outcome, a continuous audit-feedback process would be recommendable in this setting.

This study has demonstrated that perinatal audit was feasible and potentially beneficial at hospital level in Rwanda, where previously it was non-existent. A large number of factors related to the three-delays model and underlying perinatal mortality was reviewed by an audit group formed by two obstetricians and two paediatricians. Thus, perinatal audit could even contribute to improved collaboration between providers of maternal and newborn care. Our analysis also showed that the perinatal audit could further strengthen the current national guidelines by linking this audit to the audits already in place for maternal, neonatal, and child mortality.

The need to reduce perinatal mortality calls for multilevel action to address the common delays affecting the timing of access to appropriate care during pregnancy and childbirth. This entails the strengthening of maternal education on the continuum of care across all stages of the life cycle [44]. Education on pregnancy and childbirth-related issues may increase awareness about danger signs and gradually break down the sociocultural barriers that may negatively influence health-seeking behaviour. Improved knowledge on reproductive health may also prevent women from relying on traditional healers and other unqualified providers as well as from the use of harmful practices such as the intake of traditional medicines [34, 35].

Special consideration should also be given to targeted interventions for vulnerable groups, including poor households and women living without partners, which, critically, 
may lack money or access to other assistance. Strategies encouraging the practice of making savings during the course of pregnancy and foreseeing difficulties that may hamper the access to care, such as the lack of insurance and transport fees, may also be of great interest for those involved in birth preparedness and other necessary care. of the proportion of women who attended at least one antenatal care visit in this population was low (94\%) compared to the national average (99\%) [12]. Thus, an in-depth investigation is needed to provide further understanding of the causes of non-compliance to the recommended antenatal care.

In 2004, Rwanda endorsed the road-map strategy for accelerating the reduction of maternal and neonatal morbidity and mortality [45]. This strategy is based on three pillars, including emergency obstetric and newborn care, delivery by skilled birth attendants, and family planning. The mechanisms for the operationalizing of this strategy need to be strengthened to ensure that these packages can be successfully scaled up. Particular attention should be given to early detection and proper management of highrisk pregnancies, compliance with referral policies to avoid unnecessary delays, improvement of labour and fetal monitoring, and timely decision for lifesaving interventions as well as birth preparedness. Incorporating the autopsy among the routine procedures and practices also proves to be essential for improving knowledge about the causes of perinatal deaths.

Additional efforts are required to improve the skills of staff involved in the management of the mother-newborn dyad through regular training and to ensure the availability of infrastructure and equipment. Strengthening of the supervision and motivation of staff needs to be addressed through the ongoing performance-based financing approach, among other strategies. Improving vital information on perinatal health and mortality may also serve as a basis for better planning for improved survival. Ultimately, to achieve positive and sustainable results, the collaboration within and between institutions in both public and private sectors should be emphasized.

\section{Conclusions}

Perinatal audit combined with the three-delays model were applied to further understand contextual factors related to perinatal deaths occurring among delivering women in a low-income setting. The delays in careseeking, followed by suboptimal care factors, were the most frequent barriers to accessing appropriate care. The delays in reaching health facilities were less common. A large number of potentially avoidable deaths were found among fresh stillbirths and early neonatal deaths and were related to modifiable factors. A multifaceted approach, including effective strategies such as the road map, for accelerating the reduction of maternal and newborn morbidity and mortality, especially targeting single mothers or those with no insurance, needs to be strengthened for better perinatal survival. This suggests the need for the improvement of intersectoral and multilevel collaboration among healthcare providers and other stakeholders.

\section{Additional files}

Additional file 1: Case study A. (DOC $22 \mathrm{~kb}$ )

Additional file 2: Case study B. (DOC $22 \mathrm{~kb}$ )

Additional file 3: Case study C. (DOC 23 kb)

\section{Abbreviations}

AIDS: Acquired Immune Deficiency Syndrome; DH: District hospital; HIV: Human immunodeficiency virus; TRH: Tertiary referral hospital

\section{Acknowledgements}

The authors would like to thank Dr. Théobald Hategekimana and Dr. Patrick Adam Mulindwa, the directors of the hospitals involved in the study, for their permission to conduct the fieldwork. The authors would also like to acknowledge mothers who participated in the study for their consent. The authors extend their gratitude to the data collectors, the audit group, and the staff of the two hospitals for their contribution and support.

\section{Funding}

This study was funded by the Swedish International Development Cooperation Agency (Sida) within a framework of research collaboration between Uppsala University and the University of Rwanda.

\section{Availability of data and materials}

The data that support the findings of the current study are available from the corresponding author on reasonable request.

\section{Authors' contributions}

BE and LÅP conceived of the study with input from AM and CB. AM wrote the protocol that was edited by $B E, L A \AA P$ and $C B$. AM supervised the fieldwork and participated in the audit group together with PM. AM analysed the data and drafted the manuscript with substantial input from BE, $L \AA P, C B$, and JP. All authors read and approved the final version of the manuscript.

\section{Competing interests}

PM is the director of one of the hospitals involved in the study.

\section{Consent for publication}

Consent to publish social and clinical details of the babies included in the current study was obtained from their mothers. Written consent was also received from each of the three women featured in the three case studies reported in the current study. This documentation can be made available to the Editor if requested, and must be treated confidentially.

\section{Ethics approval and consent to participate}

Ethical approval for this study was granted by the Rwanda National Ethics Committee (ethics approval no. 086 RNEC/2012, Kigali, Rwanda). Informed consent was sought and received from all women whose babies were included in the study.

\section{Publisher's Note}

Springer Nature remains neutral with regard to jurisdictional claims in published maps and institutional affiliations.

\section{Author details}

${ }^{1}$ Paediatric and Child Health Department, University of Rwanda, Kigali, Rwanda. 'Department of Women's and Children's Health, International Maternal and Child Health (IMCH), Uppsala University, Akademiska Sjukhuset, Uppsala SE-751 85, Sweden. ${ }^{3}$ Center for International Health, Education, and Biosecurity (CIHEB), Institute of Human Virology, University of Maryland, 
School of Medicine MGIC-Rwanda, KG, 6 AV no 22, Kigali, Rwanda. ${ }^{4}$ Muhima District Hospital, Kigali, Rwanda.

\section{Received: 22 September 2015 Accepted: 3 March 2017 Published online: 11 March 2017}

\section{References}

1. Cousens S, Blencowe H, Stanton C, Chou D, Ahmed S, Steinhardt L, et al. National, regional, and worldwide estimates of stillbirth rates in 2009 with trends since 1995: a systematic analysis. Lancet. 2011;377(9774):1319-30.

2. Lozano R, Wang H, Foreman KJ, Rajaratnam JK, Naghavi M, Marcus JR, et al. Progress towards Millennium Development Goals 4 and 5 on maternal and child mortality: an updated systematic analysis. Lancet. 2011:378(9797):1139-65.

3. World Health Organization. ICD-10: International Statistical Classification of Diseases and Related Health Problems: $10^{\text {th }}$ revision. Volume 2: Instruction Manual. Geneva: WHO; 2004. http://www.who.int/classifications/icd/ ICD10Volume2_en_2010.pdf. Accessed 13 Feb 2017.

4. Richardus JH, Graafmans WC, Verloove-Vanhorick SP, The EuroNatal International Audit Pane, The EuroNatal Working Group. Differences in perinatal mortality and suboptimal care between 10 European regions: results of an international audit. BJOG. 2003;1 10:97-105.

5. Dunn PM, Mcilwaine G. Perinatal audit: a report produced for the european association of perinatal medicine. Carnforth: The Parthenon Publishing Group, Ltd. 1996.

6. Ivers N, Jamtvedt G, Flottorp S, Young JM, Odgaard-Jensen J, French SD, et al. Audit and feedback: effects on professional practice and healthcare outcomes. Cochrane Database Syst Rev. 2012;6:CD000259.

7. Pattinson R, Kerber K, Waiswa P, Day LT, Mussell F, Asiruddin SK, et al. Perinatal mortality audit: counting, accountability, and overcoming challenges in scaling up in low- and middle-income countries. Int J Gynaecol Obstet. 2009;107 Suppl 1:S113-21.

8. Thaddeus S, Maine D. Too far to walk: maternal mortality in context. Soc Sci Med. 1994;38(8):1091-110.

9. Mbaruku G, van Roosmalen J, Kimondo I, Bilango F, Bergstrom S. Perinatal audit using the 3-delays model in western Tanzania. Int J Gynaecol Obstet. 2009;106(1):85-8

10. Waiswa P, Kallander K, Peterson S, Tomson G, Pariyo GW. Using the three delays model to understand why newborn babies die in eastern Uganda. Trop Med Int Health. 2010;15(8):964-72.

11. Republic of Rwanda Ministry of Health. Rwanda Annual Health Statistics Booklet 2012. Kigali: MOH; 2012. http:/www.moh.gov.rw/fileadmin/templates/MOHReports/MOH_Booklet 2012 final_September_2013.pdf. Accessed 14 Feb 2017.

12. National Institute of Statistic of Rwanda (NISR) [Rwanda], Ministry of Health (MOH) [Rwanda], and ICF International. Rwanda Demographic and Health Survey 2014-15: Key Indicators. Rockville, MD: NISR, MOH, and ICF International; 2015. http://moh.gov.rw/fileadmin/templates/Narattive_ Report/Rwanda_DHS_2014-15_KIR.pdf. Accessed 14 Feb 2017.

13. Republic of Rwanda Ministry of Health: Annual Report July 2009-June 2010 Kigali, Rwanda: MOH; 2010. http://www.moh.gov.rw/fileadmin/templates/MOHReports/Final-MoH-annual-report-July-2009-June-2010.pdf. Accessed 14 Feb 2017.

14. Republic of Rwanda Ministry of Health. Annual Report July 2010-June 2011. Kigali: MOH; 2011. http://www.moh.gov.rw/fileadmin/templates/MOHReports/MoH-annual-report-2010-2011.pdf. Accessed 14 Feb 2017.

15. World Health Organization. Neonatal and Perinatal Mortality. Country, Regional and Global Estimates. Geneva: WHO; 2006. http://whqlibdoc.who. int/publications/2006/9241563206_eng.pdf. Accessed 14 Feb 2017.

16. Saleem S, McClure EM, Goudar SS, Patel A, Esamai F, Garces A, et al. A prospective study of maternal, fetal and neonatal deaths in low- and middleincome countries. Bull World Health Organ. 2014:92(8):605-12.

17. Musafili A, Essén B, Baribwira C, Selling KE, Persson LA. Social equity in perinatal survival: a case-control study at hospitals in Kigali, Rwanda. Acta Paediatr. 2015;104(12):1233-40

18. World Health Organization. Safe motherhood needs assessment. Geneva: WHO; 2001. http://whalibdoc.who.int/hq/2001/WHO_RHT_MSM_96.18_Rev. 1_Pt.1.pdf?ua=1. Accessed 14 Feb 2017

19. Lawn JE, Blencowe H, Pattinson R, Cousens S, Kumar R, Ibiebele I, et al. Stillbirths: Where? When? Why? How to make the data count? Lancet. 2011; 377(9775):1448-63

20. Rööst M, Altamirano VC, Liljestrand J, Essén B. Priorities in emergency obstetric care in Bolivia-maternal mortality and near-miss morbidity in metropolitan La Paz. BJOG. 2009;116(9):1210-17.
21. MRC Research Unit for Maternal and Infant Health Care Strategies, PPIP Users, Saving Babies Technical Task Team. Saving Babies 2003-2005: Fifth Perinatal Care Survey of South Africa. http://bettercare.co.za/wp-content/ uploads/2013/01/Saving-Babies-2003-2005.pdf. Accessed 14 Feb 2017.

22. Merali HS, Lipsitz S, Hevelone N, Gawande AA, Lashoher A, Agrawal P, Spector J. Audit-identified avoidable factors in maternal and perinatal deaths in low resource settings: a systematic review. BMC Pregnancy Childbirth. 2014;14:280.

23. Essén B, Bödker B, Sjöberg N-O, Langhoff-Roos J, Greisen G, et al. Are some perinatal deaths in immigrant groups linked to suboptimal perinatal care services? BJOG. 2002;109:677-82.

24. Lawn JE, Yakoob MY, Haws RA, Soomro T, Darmstadt GL, Bhutta ZA. 3.2 million stillbirths: epidemiology and overview of the evidence review. BMC Pregnancy Childbirth. 2009;9 Suppl 1:S2

25. Langhoff-Roos J, Larsen S, Basys V, Lindmark G, Badokynote M. Potentially avoidable perinatal deaths in Denmark, Sweden and Lithuania as classified by the Nordic-Baltic classification. BJOG. 1998:105(11):1189-94.

26. Kidanto $\mathrm{HL}$, Massawe $S N$, Nystronr L, Lindmark $G$. Analysis of perinatal mortality at a teaching hospital in Dar es salaam, Tanzania, 1999-2003. Afr J Reprod Health. 2006;10(2):72-80.

27. de Reu P, Van Diem M, Eskes M, Oosterbaan H, Smits L, Merkus H, Nijhuis J. The Dutch perinatal audit project: a feasibility study for nationwide perinatal audit in the Netherlands. Acta Obstet Gynecol Scand. 2009; 88(11):1201-08

28. Hinderaker SG, Olsen BE, Bergsjo PB, Gasheka P, Lie RT, Havnen J, Kvale G. Avoidable stillbirths and neonatal deaths in rural Tanzania. BJOG. 2003; 110(6):616-23.

29. Kidanto HL, Mogren I, van Roosmalen J, Thomas AN, Massawe SN, Nystrom L, Lindmark G. Introduction of a qualitative perinatal audit at Muhimbili National Hospital, Dar es Salaam, Tanzania. BMC Pregnancy Childbirth. 2009:9:45.

30. Nakibuuka VK, Okong P, Waiswa P, Byaruhanga RN. Perinatal death audits in a peri-urban hospital in Kampala, Uganda. Afr Health Sci. 2012;12(4):435-42.

31. El Amin S, Langhoff-Roos J, Bodker B, Bakr AA, Ashmeig AL, Ibrahim SA, Lindmark G. Introducing qualitative perinatal audit in a tertiary hospital in Sudan. Health Policy Plan. 2002;17(3):296-303.

32. De Lange TE, Budde MP, Heard AR, Tucker G, Kennare R, Dekker GA. Avoidable risk factors in perinatal deaths: a perinatal audit in South Australia. Aust N Z J Obstet Gynaecol. 2008:48(1):50-7.

33. Bapat U, Alcock G, More NS, Das S, Joshi W, Osrin D. Stillbirths and newborn deaths in slum settlements in Mumbai, India: a prospective verbal autopsy study. BMC Pregnancy Childbirth. 2012;12:39.

34. Ezugwu EC, Onah HE, Ezegwui HU, Nnaji C. Stillbirth rate at an emerging tertiary health institution in Enugu, southeast Nigeria. Int I Gynaecol Obstet. 2011;115(2):164-6.

35. Mukasa PK, Kabakyenga J, Senkungu JK, Ngonzi J, Kyalimpa M, Roosmalen VJ. Uterine rupture in a teaching hospital in Mbarara, western Uganda, unmatched case- control study. Reprod Health. 2013:10:29.

36. Say L, Raine R. A systematic review of inequalities in the use of maternal health care in developing countries: examining the scale of the problem and the importance of context. Bull World Health Organ. 2007;85(10):812-9.

37. Knight HE, Self A, Kennedy SH. Why are women dying when they reach hospital on time? A systematic review of the 'third delay'. PLoS One. 2013; 8(5):e63846.

38. Moyer CA, Mustafa A. Drivers and deterrents of facility delivery in subSaharan Africa: a systematic review. Reprod Health. 2013:10:40.

39. Påfs J, Musafili A, Binder-Finnema P, Klingberg-Allvin M, Rulisa S, Essén B. Beyond the numbers of maternal near-miss in Rwanda - a qualitative study on women's perspectives on access and experiences of care in early and late stage of pregnancy. BMC Pregnancy Childbirth. 2016;16:257.

40. Weiner R, Ronsmans C, Dorman E, Jilo H, Muhoro A, Shulman C. Labour complications remain the most important risk factors for perinatal mortality in rural Kenya. Bull World Health Organ. 2003;81(8):561-6.

41. Esscher A, Binder-Finnema P, Bødker B, Högberg U, Mulic-Lutvica A, Essén B. Suboptimal care and maternal mortality among foreign-born women in Sweden: maternal death audit with application of the 'migration three delays' model. BMC Pregnancy Childbirth. 2014;14:141.

42. Jansone M, Lazdane G. Audit of perinatal deaths in a tertiary level hospital in Latvia (1995-1999) using the Nordic-Baltic perinatal death classification: evidence of suboptimal care. J Matern Fetal Neonatal Med. 2006;19(8):503-7.

43. Farquhar C, Sadler L, Masson V. Beyond the numbers: reporting potentially avoidable perinatal deaths. BJOG. 2012;119(3):381-2. 
44. Kerber KJ, de Graft-Johnson JE, Bhutta ZA, Okong P, Starrs A, Lawn JE. Continuum of care for maternal, newborn, and child health: from slogan to service delivery. Lancet. 2007;370(9595):1358-69.

45. de Bernis L, Wolman Y. Maternal and Newborn Health: National Plans (Road Map) Assessment. African MNH Road Maps Assessment Report. New York: UNFPA; 2009. https://www.unfpa.org/webdav/site/global/shared/ documents/publications/mnh_roadmaps.pdf. Accessed 14 Feb 2017.

Submit your next manuscript to BioMed Central and we will help you at every step:

- We accept pre-submission inquiries

- Our selector tool helps you to find the most relevant journal

- We provide round the clock customer support

- Convenient online submission

- Thorough peer review

- Inclusion in PubMed and all major indexing services

- Maximum visibility for your research

Submit your manuscript at www.biomedcentral.com/submit
Biomed Central 